\title{
The MING-T approach to multi-standard network convergence
}

\author{
Norman Hendrich \\ Department of Informatics \\ University of Hamburg, Germany \\ hendrich@informatik.uni-hamburg.de
}

\author{
Jianwei Zhang \\ Department of Informatics \\ University of Hamburg, Germany \\ zhang@informatik.uni-hamburg.de
}

\begin{abstract}
This paper describes a novel research approach for multi-standard network convergence, integrating multiple broadcast networks for high-speed download with communications networks for the uplink and feedback channel. In particular, we study the integration of DVB-H and the Chinese digital television standard DTMB, with interaction provided via GSM/UMTS and WLAN.

Our software architecture for the integrated network relies on an integrated middleware framework that provides a common interface for video-streaming and IP-datacast via the different underlying networks. Unified service-information provides the user with ESG information for all available networks, and supports the cell- and inter-network handover algorithms on the mobile terminals. We describe the use-cases selected for the validation of the middleware, and the testbed that will be used for prototyping and demonstration of the overall system.
\end{abstract}

\section{Keywords}

Mobile digital television, converged networks, scalable video coding, DVB, DTMB

\section{INTRODUCTION}

Mobile users increasingly rely on access to communication and multimedia services anytime and anywhere, and only a combination of the various cellular $(2 \mathrm{G} / 2.5 \mathrm{G} / 3 \mathrm{G})$ communication networks, IP-based wireless networks (WLAN, WiMAX), and the digital broadcast networks (e.g. DAB, DVB, DTMB, [1]) will provide a balanced overall system capable to keep up with the customers' demands. In this so-called converged network, the different individual networks complement each other and integrate to provide a common high-performance and ubiquitous communication infrastructure.

This paper presents an overview of the joint European and Chinese project MING-T (Multistandard Integrated Network Convergence for Global Mobile and Broadcast Technologies, www.ming-t.eu), funded by the EC as part of the FP6-IST programme. The goal of

Mobimedia 2008 July 7-9, 2008, Oulu, Finland. Copyright 2008 ICST ISBN 978-963-9799-25-7 DOI 10.4108/ICST.MOBIMEDIA2008.3864

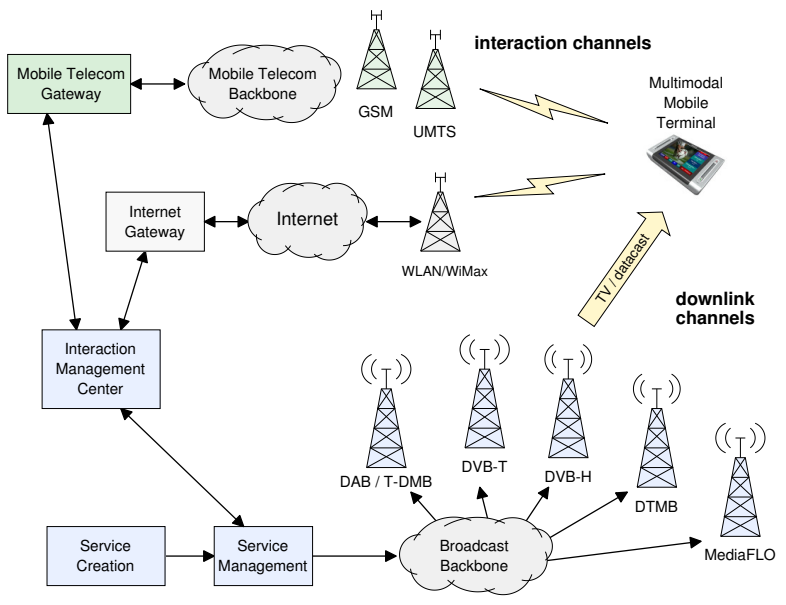

Figure 1: Overview of the converged multi-standard network including broadcast (DVB, DTMB), mobile communication (2G/3G), and WLAN networks.

the project is to research, develop, prototype, and validate an architecture that provides seamless access in converged digital broadcasting and mobile communication networks. We consider the representative broadcast standards of Europe and China, especially DVB-H $[2,3]$ and DTMB [4]. Considering the ongoing progress in microelectronics and signal-processing, we envision that multistandard broadcast receivers will be an integral component of future cellphones and PDAs.

Figure 1 illustrates the wireless universe underlying our model of the converged network. Audio, video, multimedia, and general IPdata is transmitted to the users via multiple broadcast networks. The cellular networks provide the usual voice and data services, but also the uplink channel that allows the user to request services from the broadcast networks. Of course, WLAN is considered as an alternative bidirectional communication channel. Typical bandwidths vary from roughly $10 \mathrm{kbps}$ for GSM, $384 \mathrm{kbps} .2 \mathrm{Mbps}$ for UMTS, 3.2 Mpbs per DVB-H channel, up to $30 \mathrm{Mbps}$ for a DVB-T or DTMB channel (in $8 \mathrm{MHz}$ bandwidth), and $54 \mathrm{Mbps}$ for IEEE 802.11g.

The rest of this paper is organized as follows. First, section 2 summarizes previous research and some observations regarding both the technical and the business aspects of the converged networks. Some key aspects of the Chinese DTMB broadcast standard and 
the compatibility with DVB are sketched in section 3. Next, section 4 describes the set of use-cases and scenarios that will form the basis of the project software development. The architecture of the planned middleware that forms the core of the project research is presented in section 5. Finally, in section 6 we describe the planned testbed that will be used for the experiments and the demonstrations.

\section{RELATED WORK}

Due to the obvious potential, all major aspects of the network convergence have recently attracted a lot of intensive research.

Cellular and Handover: The seamless horizontal handover of an ongoing phone-call between different base stations has been a key feature of the GSM networks from the start [8]. With the introduction of the $3 \mathrm{G}$ networks, this was extended to the so-called vertical handover between the $2 \mathrm{G}$ and $3 \mathrm{G}$ networks [9]. On the other hand, support for IP-based multimedia has been a recent evolution of the cellular networks [10], as has been the integration and handover between cellular and WLAN [11].

Mobile IP: The increasing popularity of wireless local-area networks has spawned a lot of research into roaming and handover between different WLAN cells [12]. Roaming in IPv4 networks is difficult, because the unique IP addresses of the nodes are allocated based on the subnets that the nodes belong to. A moving node changes its subnet, and therefore also its IP address. Several solutions have been proposed, including Mobile-IP [13] and Mobile-IPv6 [14].

Broadcast and Cellular: In recent years, several groups have proposed to complement a digital broadcast channel with an interaction channel via a cellular network. Proposed combinations include DVB-T with GSM [15] or GPRS [16], and DVB-T with UMTS [17]. The integration with WLAN has also been studied and demonstrated for DTMB [18].

QoS and Seamless Handover: While a best-effort strategy is often sufficient for data-transfers, most multimedia services require some sort of quality-of-service (QoS) guarantees. Users might tolerate drop-outs of a few $100 \mathrm{~ms}$ during a phone-call, but even a few dropped video-frames are very noticeable. Therefore, the duration of a seamless (unnoticed) handover is critical. A recent paper concluded that a handover time of about $100 \mathrm{~ms}$ was possible in Mobile-IPv6, [20]. The inter-cell handover in DVB-H networks has also attracted a lot of interest recently [21].

\section{DTMB}

While details of the ATSC, DVB, and ISDB-T digital television standards are widely known (see [1] for an overview), the Chinese standard for terrestrial digital television, called DTMB, was published only recently in August 2006 [4].

DTMB specifies two main operating modes. The first mode is a multi-carrier system developed at Tsinghua University in Beijing, while the second mode is a single-carrier system proposed by Jiaotong University in Shanghai. The system offers payload data-rates from 4.8 Mbps to $32 \mathrm{Mbps}$, supports stationary and mobile reception, and can be used in single-frequency networks (SFN).

While DVB-T and DVB-H rely on the well-known coded orthogonal frequency division multiplexing modulation with cyclic prefixes (CP-COFDM), the multi-carrier system in DTMB uses a new scheme called time domain synchronous OFDM (TDS-OFDM), which inserts pseudo-noise sequences into the guard intervals. This allows the receiver to quickly synchronize with the signal and to estimate the subcarrier channel characteristics at the same time.

Instead of the concatenated RS and convolution codes used in DVB-T, DTMB employs forward error correction based on concatenated BCH and LPDC codes, resulting in superior error correction and an improved sensitivity. The system is claimed to provide a bit error rate of less than $10^{-10}$ in typical receiver conditions, and supports high-speed mobile reception up to $130 \mathrm{~km} / \mathrm{h}$. Finally, DTMB uses a hierarchical framing structure that is kept synchronous to real time. This provides a precise timebase to the receiver and supports automatic wake-up and power-saving functions.

While deployment of DTMB has started in 2008, many aspects of the system are still under discussion and final standards have not been published yet. However, it is known that the architecture will use the MPEG-2 transport stream (TS) and IP-encapsulation for data services. Of course, the TS also carries service information (SI) and electronic service guide (ESG) tables similar to DVB-T.

Despite the differences outlined above, dual-mode receiver chips that support both DVB and DTMB are certainly feasible. An initial analysis of a possible hardware structure shows that key building-blocks like the 8192/3780-points FFT module required for the OFDM in DVB/DTMB can be shared [19]. However, no dualmode receivers have been announced yet, and we will use two separate receivers for the project demonstrator.

\section{SERVICE SCENARIOS}

To guide the research, our project has identified and selected a set of eight typical use-cases for the integrated network [25].

Unified service discovery: The first scenario is concerned with the service discovery and service description in the converged multistandard network. Basically, we propose to broadcast extended service information that carries information not only for the currently selected channel, but about all networks that cover the users' location. This is achieved by embedding suitable information tables into the MPEG-2 transport stream [22]. The obvious extension of the network information table (NIT) [23] allows us to broadcast frequency and synchronization information about other networks, which also facilitate roaming and handover decisions. An extended electronic service guide (ESG) provides the user with information about programs on all networks.

Access to multiple networks: The second scenario allows the user to watch a program via multiple networks. After discovering an interesting service via the extended ESG, the user is presented with all networks that offer the service and can then select the network and provider. Of course, the assumption that particular content is available at the same time via different networks might be challenged. However, it will be true in many cases. Typical examples are highprofile international or national sports events (Olympic games, soccer worldcup, etc.), or cultural events like concerts and festivals. In such cases, all networks are expected to have live coverage, with either the same or at least very similar programming (e.g. live coverage of the same event, but using different camera positions). Another example is provided by news-channels or music videos. Here, different networks can be expected to distribute different but similar content, and users might appreciate the extra choice. 
Protected content: Our third use-case is the access to protected broadcast services, where the user is given the option for pay per view. The idea here is to use the mobile communication network or WLAN for customer authentication and authorization. This goes beyond the traditional model already in use today, where users get access to encrypted content on the basis of pre-paid or subscription contracts, but without real-time interaction with the network operators.

Video-on-demand: The extension of the above use-case to videoon-demand downloads via the broadcast networks forms our fourth scenario. Again, payment is handled via the mobile communication or WLAN networks. We will study both real-time streaming of the video data and asynchronous download scenarios, where the data is stored on the mobile terminal until the user watches the program. We will also study a mechanism where the network tries to collect requests from several users before starting the transmission. In this case, the mobile terminals will be notified via the ESG about the upcoming session. A mobile terminal with separate broadcast receivers might be able to increase download-speed by parallel downloads from several networks.

Auxiliary data and interaction: This use-case is concerned with enriched media, where an ongoing broadcast is enhanced with auxiliary data, e.g. subtitles, hypertext documents with background information, additional multimedia, etc. The auxiliary data can be embedded into the broadcast stream, or be sent on demand via the mobile interaction network. The mobile terminal notifies the user of available auxiliary data, and renders the data as an overlay or on separate pages. The display also provides a context-sensitive userinterface that allows the customers to select specific actions, for example chatting with other users, e-commerce transactions, voting, and so on. Again, the corresponding interactive data-transfers use the uplink and feedback channel.

Broadcast service for roaming terminal: The above scenarios apply for both stationary and mobile reception. As indicated in section 2, users take roaming between mobile communication networks almost for granted. Our sixth use-case considers the roaming between several broadcasting networks, and the required authorization and payment mechanisms. For example, a user moves out of the coverage of his DVB-H home network while watching a subscription sports channel, and enters a region covered by DTMB with a similar sports channel, but differing payment options.

Scalable-coding: With a slightly different focus, our next use-case targets the analysis of scalable-coding algorithms to ensure the best image quality possible given the current receiver conditions and setup. The idea is to partition the full-quality image information into two or more layers, which are broadcast and decoded separately. The low-bitrate standalone base-layer uses a robust encoding to ensure the reception and display of a basic quality video even under adverse conditions. One or more enhancement layers use higher bitrates and more efficient (but fragile) encodings to provide additional image detail.

While scalable-coding has been an option of the well-known video standards (e.g. MPEG-2, H.263, H.264) for some time, the additional encoding complexity and the non-negligible overhead have limited its widespread use. However, the advantages of scalablecoding for mobile television are clear, and the hierarchical modulation specified for DVB-T and DVB-H provides a straightforward way to encode a robust low-bitrate base-layer and a high-bitrate enhancement-layer bitstream. Implementing scalable-coding on top of DTMB is more challenging. Our research will be based on H.264 SVC and addresses the choice of scalability (spatial, temporal, SNR, etc.), the integration into the constraints imposed by DVB-H and DTMB, and the obvious business scenarios (e.g., extra billing for the enhancement layers).

Seamless handover: The final scenario considered in the project is the automatic and seamless handover between different broadcast and communication networks. In particular, we will strive to implement and prototype the handover between DVB-H and DTMB networks, under the assumption that both networks carry the same content. The decision to switch the network will be made by the receiver based on incoming signal strength and/or bit-error rates. For video applications, the handover can be combined with the scalable-coding scenario, in order to ensure the best image quality possible and a graceful degradation under poor reception conditions.

\section{CONVERGENCE MIDDLEWARE}

Given the diversity of the current-generation communication and broadcasting networks, the question arises of how to integrate those networks into one common converged network.

Following the success of the internet, IP is generally considered to be the key element to enable communication between the different networks. In this case, the basic architecture of the converged network becomes obvious. Each individual network is connected to the common IP backbone via its own specific gateway, which translates from IP to the protocols used inside the corresponding network. The backbone itself is connected via one or more gateways to the internet.

The encapsulation and transmission of IP data has been specified for both communication networks (e.g. UMTS IMS [10]) and broadcast networks (e.g. [5], [6], [7]). The challenges resulting from user-mobility and handover, and a few solutions (e.g. MobileIP [13]), were already mentioned in section 2.

On the receiver side, we propose using a common middleware framework that provides a unified interface to the upper application layers, as shown in figure 2. The framework is divided into two separate but closely cooperating parts, one for the communication networks (on the left) and one for the several broadcast networks (on the right). Both parts are then further subdivided into several layers. Details and the API specification have recently been published by the project [26], [27].

The lowest layer forms the interface to the actual device-drivers and retrieves the raw bitstreams and status parameters like signalstrength or error-rates from the receiver hardware. The figure shows six example drivers on this layer (WLAN, GSM, UMTS, DVB-H, DTMB, T-DMB). Each adapter is coupled with an adaptation layer that converts the incoming raw data into the format expected by the convergence sublayers.

The role of the convergence sublayers is two-fold. First, this is where the incoming bitstreams are converted to IP packets for use by the upper layers. Second, the convergence sublayer is responsible for the handover between different broadcast networks, and the handover and handshake between broadcast and communication networks. 
Converged Interactive Services / User-Interface

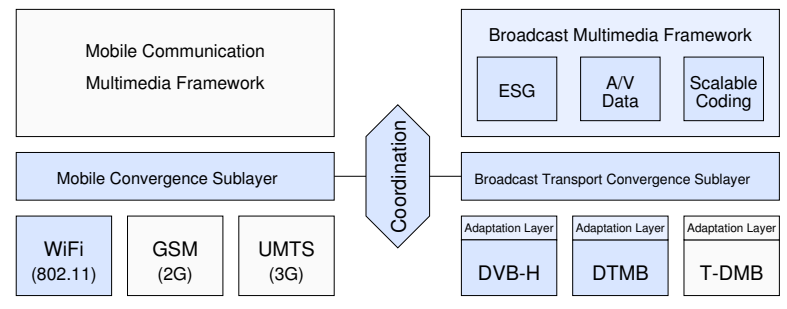

Figure 2: Block-diagram of the proposed receiver middleware software architecture.

Above the convergence sublayers are the multimedia frameworks, which provide the audio/video decoding and corresponding functions. In the context of our project, we are mostly concerned with the broadcast multimedia framework (on the right) and three of its core components. The ESG component is used to extract and parse the unified service guide information. Similarly, the $A / V$ and data component is used to extract and handle the raw MPEG-2 datapackets. A separate module is responsible for parsing and synchronizing the separate base-layer and enhancement-layer bitstreams for the scalable video coding;

The topmost layer of our receiver framework is the converged interactive services layer, which includes a prototype user-interface. This will support the use-cases listed above in section 4 . For example, we will include controls that let the user select a network manually, select whether and how to display auxiliary data together with an ongoing video, and to execute pay-per-view transactions.

The standard application software sits above the converged interactive services layer shown in figure 2 . This includes software like the program guide, web-browser, Java virtual machine, stock-market information, interactive games, etc.

\section{THE TESTBED}

The experiments to validate our software and prototypes will be carried out on the campus of Tsinghua University in Beijing. Close to the city center, the campus is located in a typical urban scenario and provides us with an ideal terrain for meaningful measurements of receiver performance. Additionally, we can share the existing network and transmitter infrastructure already in-place, including the complete testbed used for the further development of the DTMB physical-layer.

Figure 3 shows the overall architecture of the proposed testbed. Starting at the top left, the incoming A/V streams are first encoded into MPEG-2 format; the optional encoding into base-layer and enhancement-layer streams as required for scalable-coding is not shown in the figure. Parallel to the video-encoding, the IP data requested by the customers is converted to the transport stream format via an IP-encapsulator. The multi-network service information and all streams are then multiplexed into the MPEG-2 transport streams, sent to the DTMB and DVB-H encoders, and the actual transmitters. While the transmitter equipment is ready for SFN operation, this will not be used for the final demonstration to avoid the extra cost and complexity.
The broadcast signals are then received by the multistandard mobile terminal shown on the lower right. As no single-chip receivers that support both DVB-H and DTMB are available yet, a notebook computer connected to two separate DVB-H and DTMB receiver cards will be used for the experiments. The built-in WLAN provides access to the campus network, while a GSM/UMTS card connects to the communication networks. While not as portable as a dedicated device, the notebook setup is small enough to allow experiments from moving vehicles and even handheld.

The planned experiments will cover the most important aspects of the multi-standard networks. This includes the transmission and reception of video and data-streams via both DVB-H and DTMB, decoding and rendering of the integrated ESG information, and the selection of video and data-services from the ESG, including the video-on-demand scenarios with billing. Based on receiver signalstrength and error-rates, we will demonstrate both the handover between DTMB and DVB-H and the use of scalable-coding to automatically switch between the base-layer and enhancement-layer quality. All required equipment is readily available from the respective project partners.

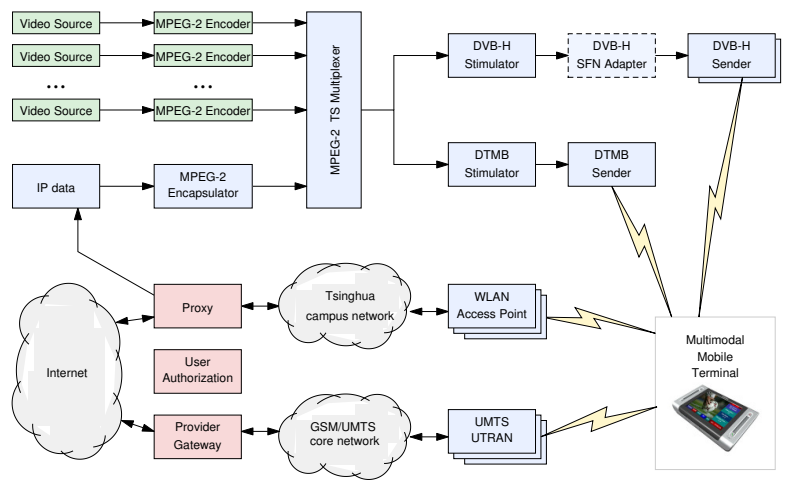

Figure 3: Block diagram of the project testbed.

Besides sharing the existing DTMB equipment, our experiments will also take advantage of the insights gained by the Tsinghua groups while developing their integrated network [18]. Our filedownload scenario will use the improved protocol stack for datatransmission on top of the raw MPEG-2 transport stream, including the new sub-network data-unit (SDNU) layer, and support for bandwidth-allocation and QoS management.

While some bit-errors due to bad channel conditions might be acceptable for real-time video and audio services, packet-loss is usually not an option for data services. In a pure broadcast network, even a very strong FEC with corresponding overhead cannot guarantee error-free delivery. In the integrated network, clients can easily use the uplink channel to request the re-transmission of lost data packets (ARQ). Under normal conditions, the number of lost packets will be low, and a NAK (not acknowledge) mechanism will be much more efficient than the tranditional TCP ACK, which acknowledges each incoming data packet. However, even a standard NAK protocol could potentially flood the feedback network, because reception errors often occur in bursts.

Therefore, the Tsinghua group has proposed and implemented an improved NAK protocol that also efficiently handles burst-errors. 
Additionally, two optimizations are introduced to reduce the volume of the re-transmitted data [18]. First, the transmitter does not retransmit a requested packet immediately, but introduces a short delay to collect and bundle requests from multiple clients. Second, data-packets are not re-transmitted individually, but rather as the bitwise XOR-sum (parity-encoded) of multiple packets requested by different receivers. As a client already has all except the single missing one of the data-packets bundled into one of those parityencoded packets, it can easily reconstruct and extract the missing packet. This optimization requires a careful tradeoff between the number of data-packets that can be merged before transmission and the delay until re-transmission.

\section{CONCLUSION}

This paper presented the concept of an integrated converged wireless network that includes multiple broadcast and communication systems. We have identified relevant use-cases and indicated how a middleware framework can be used to connect the networks and provide free or protected services to the users. We have described the demonstration testbed currently under construction on the campus of Tsinghua University in Beijing. It combines the Chinese DTMB and the European DVB-H broadcast networks with UMTS and WLAN for interaction, and will provide ample opportunity to test and improve algorithms to ensure robust and uninterrupted high-bandwidth wireless services.

\section{Acknowledgment}

The research in this paper was partly funded by the EC project MING-T (Multi-standard integrated network convergence for global mobile and broadcast technologies) under contract IST2006-045461. The authors gratefully acknowledge the contributions and discussions of all project partners.

\section{REFERENCES}

[1] Y. Wu, S. Hirakawa, U. H. Reimers, and J. Whitaker (Eds.), Global Digital Television: Technology and Emerging Services, Special Issue, Proceedings of the IEEE, vol 94, no. 1, 5-332, 2006

[2] U. Reimers, $D V B$ - The family of international standards for digital video broadcasting, 2nd. ed., Springer-Verlag, Germany, 2005

[3] G. Faria et.al., DVB-H: Digital Broadcast Services to Handheld Devices, Proc. IEEE, vol. 94, no. 1, 194-209, 2006

[4] Framing structure, channel coding and modulation for digital television terrestrial broadcasting system, GB 20600-2006, National standard of P. R. China, 2006

[5] ETSI, Digital Video Broadcasting (DVB); DVB specification for data broadcasting, EN 301192 V1.4.1, ETSI standard, 2004

[6] ETSI, IP Datacast over DVB-H: Architecture, TS 102469 V1.1.1, 2006

[7] Dimitris Kouis, Dimitris Loukatos, Kimon Kontovasilis, Geroge Kormentzas, Charalabos Skianis On the effectiveness of DVB-T for the support of IP-based services in heterogeneous wireless networks, Computer Networks 48 (2995), 57-73

[8] ETSI, Digital cellular telecommunication system - Handover procedures, TS 100527 V7.0.0, 1998

[9] ETSI, Handover requirements between UTRAN and GERAN or other radio systems TS 122129 V7.0.0, 2006
[10] Y.-B. Lin, A.-C. Pang, Y.-R. Haung, and I. Chlamtac, $A n$ All-IP Approach for UMTS Third-Generation Mobile Networks, IEEE Network, vol.16, no.5, 8-19, 2002

[11] ETSI, UMTS - 3GPP system to Wireless Local Area Network (WLAN) interworking, ETSI TS 129234 V7.5.0, 2007

[12] D. Saha, Am. Mukherjee, I. S. Misra, M.1,Chakraborty, Mobility Support in IP: A Survey of Related Protocols, IEEE Network, vol 18, no.6, 34-40, 2004

[13] C. Perkins, Ed., IP Mobility Support, IETF RFC 2002, 1996

[14] D. Johnson, C. Perkins, J. Arkko, Mobility Support in IPv6, IETF RFC 3775

[15] ETSI, DVB - Interaction channel through the Global System for Mobile Communications (GSM), EN 301195 V1.1.1, 1999

[16] C. Rauch, W. Kellerer, and P. Sties, Hybrid mobile interactive services combining DVB-T and GPRS, Proc. EPMCC 2001, 4th European Personal Mobile Communications Conference, Vienna, 2001

[17] J. Cosmas et.al., System Concept of a Novel Converging DVB-T and UMTS Mobile System, Proc. IEE\&IEEE London Communications Symposium 2002, 97-100, 2002

[18] L. Long, Z. Niu, and B. Zhu, A Hybrid DMB-T and WLAN Network for Broadband Wireless Access Services, IEEE Wireless Communications and Networking Conference (WCNC' 07), 4289-4294, Hong Kong, March 2007

[19] F. Yang, J. Song, C. Pan, H. Yang, K. Peng, J. Wang, and Z. Yang, An Initial Study on the Convergence of DVB-H and $D T M B$ in the Physical Layer, private communication

[20] R. Pries, A. Mäder, D. Staehle, M. Wiesen, On the Performance of Mobile-IP in Wireless LAN Environments, Proc. Wireless and Mobility in Next Generation Internet, LNCS 4396, pp.155-170, 2007.

[21] X. Yang, J. Väre, T.J.Owens, A Survey of Handover Algorithms in DVB- $H$, in IEEE Communications Surveys and Tutorials, Dec. 2006

[22] ISO/IEC, Information technology - Generic coding of moving pictures and associated audio information: Systems (MPEG-2), ISO/IEC standard 13818-1, 2000

[23] ETSI, Specification for Service Information (SI) in DVB systems, EN 300 460, V1.8.1, (2007)

[24] TV Anytime Forum and ETSI, TV-Anytime, part 2, phase 1 system description, TS 102 822-2 (2007)

[25] Jiangang Yu, Ed., Scenarios, Requirements, and Initial MING-T Architecture, report IST-045461 D1.1, www.ming-t.eu

[26] Yanyu Ma, Ed., MING-T: Initial middleware specification, report IST-045461 D2.1, www.ming-t.eu

[27] Yanyu Ma, Ed., MING-T: Initial API specification, report IST-045461 D2.2, www.ming-t.eu 